\title{
Inter- and intra-observer reliability of the Baumann angle of the humerus in children with supracondylar humeral fractures
}

\author{
Mauricio Silva $\cdot$ Rajeev Pandarinath $\cdot$ Eugene Farng • \\ Samuel Park • Cherlyn Caneda • Yi-Jen Fong • \\ Adam Penman
}

Received: 9 March 2009 / Accepted: 7 April 2009/Published online: 8 May 2009

(C) The Author(s) 2009. This article is published with open access at Springerlink.com

\begin{abstract}
The Baumann angle of the humerus has been commonly used as an outcome measure for supracondylar fractures in children. However, there is limited or no information about the reliability of this measurement. The purpose of this study was to determine the inter-observer reliability (IEOR) and intra-observer reliability (IAOR) of the Baumann angle of the humerus. The Baumann angle of the humerus was measured by five observers on the anteroposterior radiographs of 35 children's elbows, all of which had sustained a nondisplaced supracondylar humeral fracture. The values of IEOR and IAOR were calculated using a Pearson coefficient of correlation. Ranges of differences in the measurement of the Baumann angle of the humerus were established, and the percentage of agreement between observers was then calculated using those ranges. The Baumann angle of the humerus is a simple, repeatable and reliable measurement that can be used for the determination of the outcome of supracondylar humeral fractures in the paediatric population. An excellent IEOR was found for the measurement of the Baumann angle ( $r=0.78, p=0.0001)$. When the difference between observers in the reported measurement of the Baumann's angle was calculated to be within seven degrees of each other, at least four of the five observers agreed $100 \%$ of the time. Similarly, excellent values of IAOR were found for the measurement of the Baumann's angle $(r=0.80, p=0.0001)$. Level of evidence for this study was III.
\end{abstract}

M. Silva $(\bowtie) \cdot$ C. Caneda $\cdot$ Y.-J. Fong $\cdot$ A. Penman

Los Angeles Orthopaedic Medical Center,

2400 S Flower Street,

Los Angeles, CA 90007, USA

e-mail:msilva@laoh.ucla.edu

M. Silva $\cdot$ R. Pandarinath $\cdot$ E. Farng $\cdot$ S. Park

Orthopaedic Hospital/UCLA Department of Orthopaedics,

David Geffen School of Medicine at UCLA,

Los Angeles, CA, USA

\section{Introduction}

Supracondylar humeral fractures are common injuries in the children [1-3]. The outcome of supracondylar fractures in this population has been commonly determined by clinical and radiographic parameters including, among others, the Baumann angle of the humerus $[2,4,5]$.

The angle formed by the intersection of a line drawn down the humeral shaft axis and a line drawn along the physeal line of the lateral condyle has been commonly described as the Baumann angle of the humerus [2, 3, 6]. Dr. Ernst Baumann, while treating displaced supracondylar humeral fractures in children with closed reduction and olecranon overhead traction, demonstrated that this angle is a useful indicator of the adequacy of reduction of a displaced supracondylar humeral fracture [6]. A decreased Baumann angle suggests a residual varus angulation of the distal humerus.

Normal values of the Baumann angle in the paediatric population have been reported to range between $9^{\circ}$ and $26^{\circ}$ $[2,5,7]$. Multiple factors that could explain the wide range in the normal value of the Baumann angle have been investigated [4, 8]. However, to our knowledge, there is limited or no information about the reliability of the measurement of the Baumann angle.

The purpose of this study was to determine the interobserver reliability (IEOR) and intra-observer reliability (IAOR) of the Baumann angle of the humerus.

\section{Materials and methods}

An IRB-approved data collection study on paediatric elbow fractures is currently underway at the Orthopaedic Hospital Outpatient Medical Center. As part of this study, clinical and radiographic information is collected from patients younger than 14 years of age who were seen at the 
Orthopaedic Hospital Outpatient Medical Center and diagnosed with a supracondylar humeral fracture.

Of the entire population enrolled in the study, 35 patients with minimally displaced supracondylar humeral fractures, who were treated with cast immobilisation, and who had completely recovered the elbow range of motion at the latest follow-up (as compared to the normal, contralateral side), were included in the study. The mean age at the time of the fracture was 6.2 years (range 1.3-11.4 years, SD 2.2 years). The mean follow-up was 18.5 weeks (range 7.0-52.0 weeks, SD 11.5 weeks).

The anteroposterior radiographs of the affected elbows $(n=35)$, obtained during the latest follow-up visit, were used with the purpose of determining the IEOR and IAOR of the Baumann angle of the humerus. All radiographs were taken digitally by an experienced technician, following standard techniques. The Baumann angle was measured using the Cobb tool available in our digital radiology package (Office PACS V 3.4.0.38, Stryker Imaging, Flower Mound, TX) by drawing a line perpendicular to the longitudinal axis of the humeral shaft, and a line following the physeal line of the lateral condyle [2]. The angle between these two lines was used as the Baumann angle.

The Baumann angle of the humerus was measured on the anteroposterior radiographs of these 35 elbows by five observers, including one paediatric orthopaedic surgeon, three orthopaedic surgery residents, and one certified paediatric nurse practitioner (a total of 175 observations). All observers received specific instructions on how to measure the angle in order to ensure a standardised technique. All radiographs were presented to the observers in a random fashion, blinding any identifying patient information. All five observers repeated their measurements on all 35 radiographs in a similar fashion (a total of 175 observations), after a period of at least two weeks from the initial assessment, to avoid recall bias. All five observers were blinded to the results of their first evaluation. In order to determine the IEOR of the Baumann angle of the humerus, the measurements obtained in both data-collection sessions were included (a total of 350 observations). For the determination of IAOR, the results of each one of the datacollection sessions (175 observations each) were compared.

Blinding and randomisation of the patients, as well as collection of the data, was performed by an independent investigator not associated with the evaluation process.

The statistical analysis was performed using STATA (Stata, College Station, TX). The Baumann angles obtained in each one of the data-collection sessions were compared using a paired $t$-test. A $p$ value of less than 0.05 was considered significant. In order to determine IEOR and IAOR, and because the measurements were on a numerical scale, a Pearson coefficient of correlation was used. Correlations from 0 to 0.25 indicated poor reliability. Those from 0.25 to 0.50 indicated a fair degree of reliability. Those from 0.5 to 0.75 indicated good reliability, and those greater than 0.75 indicated excellent reliability. The frequency of agreement (as a percentage) between observers was determined using the raw data. Taking into consideration that a small difference in measurements might not represent a meaningful disagreement between observers, ranges of differences in the measurement of the Baumann angle were established, and the percentage of agreement between observers was then calculated using those ranges.

\section{Results}

During the first data-collection session (175 observations), the mean Baumann angle of the humerus was found to be
Fig. 1 The inter-observer reliability (IEOR) of the measurement of the Baumann angle was found to be excellent $(r=0.78, p=0.0001)$. Note the linear relationship between the measurements of the Baumann angle of the humerus by different observers

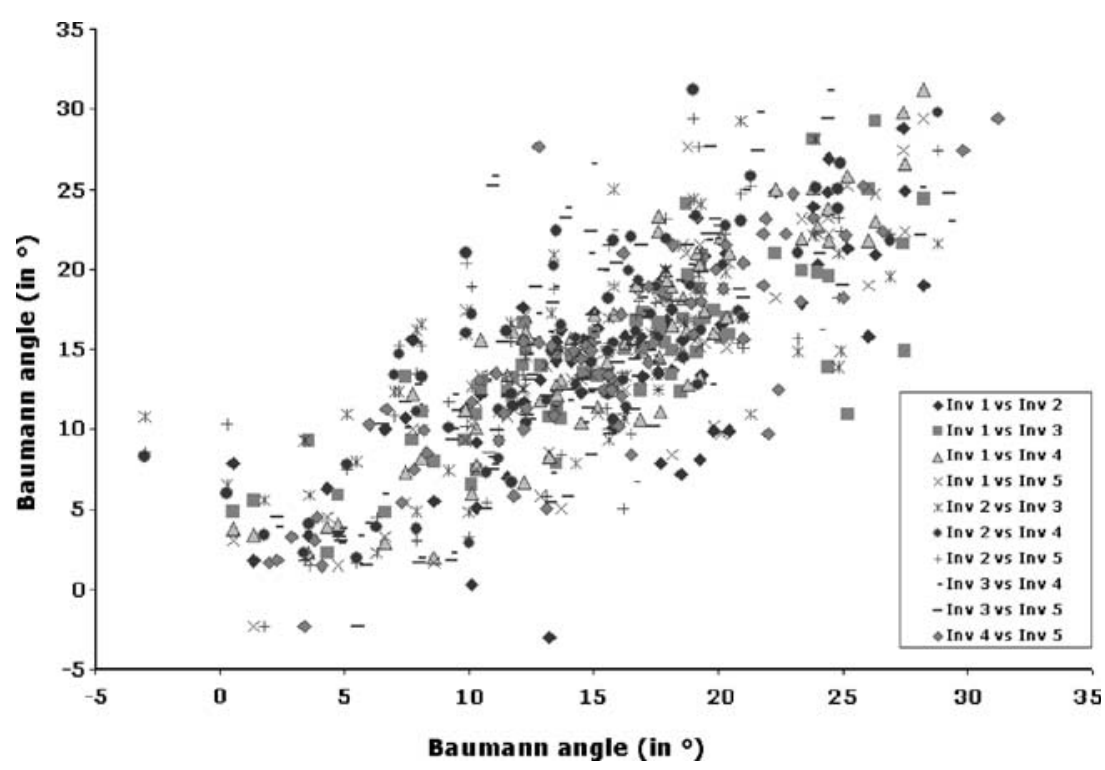


Fig. 2 When the difference was calculated to be within 7 degrees of each other, at least four of the five observers agreed $100 \%$ of the time

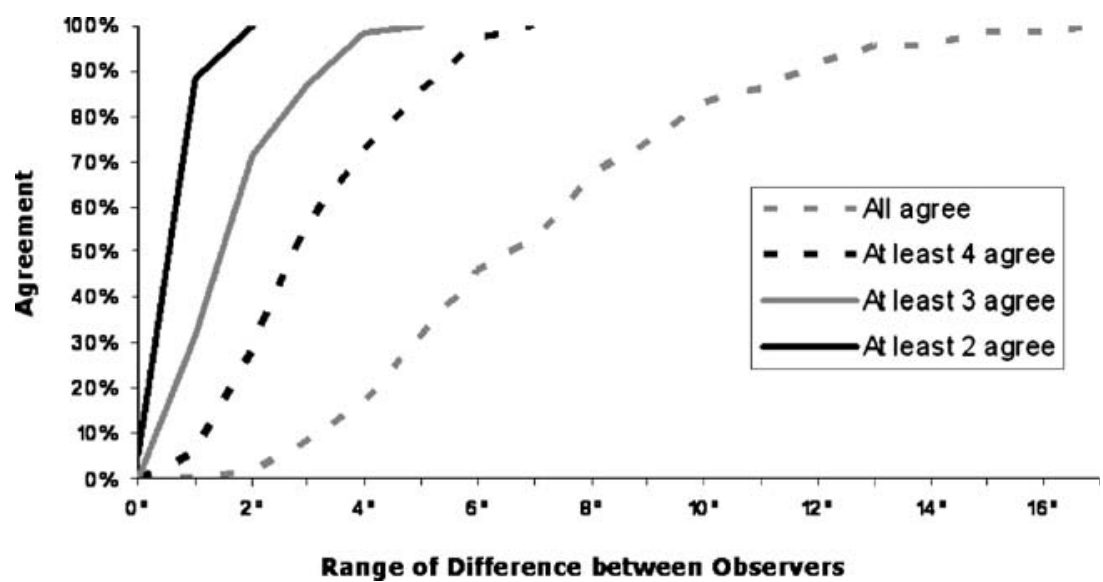

$14.7^{\circ}$ (range $-3.0^{\circ}$ to $29.8^{\circ}$, SD $6.4^{\circ}$ ). This was not significantly different from the data obtained during the second data-collection session (more than two weeks apart, 175 observations) (mean $15.2^{\circ}$, range $-2.3^{\circ}$ to $31.2^{\circ}$, $\mathrm{SD}$ $\left.6.3^{\circ}\right)(p=0.78)$.

An excellent IEOR was found for the measurement of the Baumann angle $(r=0.78, p=0.0001)$ (Fig. 1). When the difference between observers in the reported measurement of the Baumann's angle was calculated to be within $5^{\circ}$ of each other, at least three of the five observers agreed $100 \%$ of the time (Fig. 2). When this difference was calculated to be within $7^{\circ}$ of each other, at least four of the five observers agreed $100 \%$ of the time (Fig. 2).

Similarly, excellent values of IAOR were found for the measurement of the Baumann's angle $(r=0.80, p=0.0001)$ (Fig. 3). When the difference between the first and second measurement of the Baumann's angle of the humerus (for each one of the observers) was calculated to be within $7^{\circ}$, the mean agreement between the two observations was found to be $91.4 \%$.

Fig. 3 The intra-observer reliability (IAOR) of the measurement of the Baumann angle was found to be excellent $(r=0.80, p=0.0001)$

\section{Discussion}

The outcome of supracondylar humeral fractures in the paediatric population has been commonly assessed by clinical and radiographic parameters, including the Baumann angle of the humerus [5, 9-15]. To our knowledge, there is limited or no information about the reliability of this radiological measurement.

Normal values of Baumann angle in the paediatric population have been reported to range between $9^{\circ}$ and $26^{\circ}[2,5,7]$. In an attempt to explain the wide range in the normal value of the Baumann angle, multiple factors that could affect both the anatomy of the distal portion of the humerus as well as the physical measurement of the Baumann angle have been investigated. Keenan and Clegg, after examining the radiographs of 577 paediatric elbows, performed an analysis of variance that suggested that neither age, gender, nor side measured affected the Baumann angle of the humerus [8]. Camp et al. showed that the Baumann angle of the humerus varies $6^{\circ}$ for every

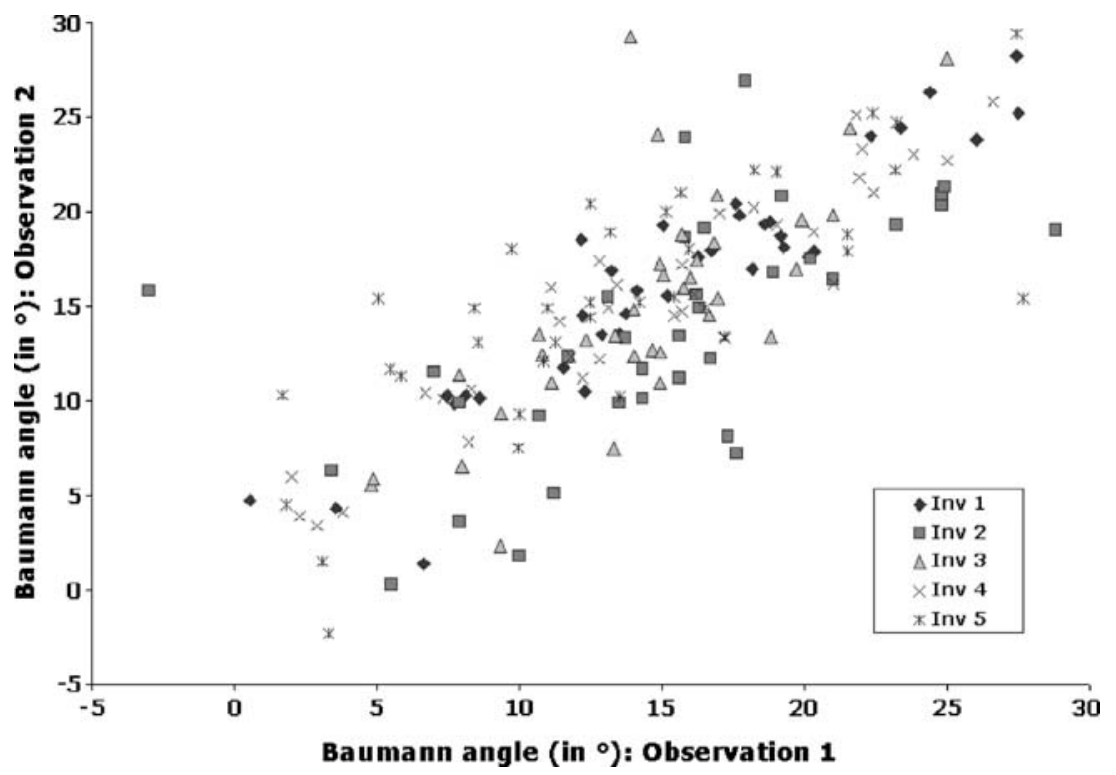


$10^{\circ}$ of humeral rotation on the anteroposterior radiograph of the elbow [4]. Inter-observer variability could also, at least in theory, play a role in the observed variability of the measurement of the Baumann angle of the humerus. However, the inter- and intra-observer reliability values for the measurement of the Baumann angle of the humerus, to our knowledge, have not been previously determined.

This study demonstrates that the Baumann angle of the humerus is a highly reliable measurement, with excellent inter-observer and intra-observer reliability values $(r=0.78$ and $r=0.80$, respectively). In most instances, measurements of the Baumann angle of the humerus (by different observers, as well as by a single observer on multiple occasions) were within $7^{\circ}$ of each other. Therefore, a difference of up to $7^{\circ}$ in the measurement of the Baumann angle (by a single or multiple observers) should be considered to be within the normal error of the measurement.

Taking into consideration that the Baumann angle of the humerus is used on a regular basis as a predictor to determine the outcome of paediatric supracondylar humeral factors, the results of this study have significant clinical implications. By establishing the inter- and intra-observer variability of the Baumann angle of the humerus, and therefore the normal error of the measurement, clinicians can better determine the outcome of paediatric supracondylar humeral fractures. Moreover, the results obtained in different series dealing with the outcome of such fractures can be compared with greater accuracy.

In this study, excellent values of inter- and intra-observer reliability were found for the measurement of the Baumann angle of the humerus, despite the fact that the level of expertise was different for at least three of the five observers. In clinical research, an ideal outcome measure is one that is simple, repeatable, and that can be applied universally by a wide range of individuals, without regard to their level of expertise. The results of this study suggest that the Baumann angle of the humerus fulfills those criteria. Therefore, the measurement of the Baumann angle of the humerus should be considered when performing outcome research on paediatric supracondylar humeral fractures.

The purpose of this study was not to determine the normal values of the Baumann angle of the humerus in paediatric patients with supracondylar humeral fractures; instead, our purpose was to evaluate the repeatability of the measurement of the Baumann angle. For that reason, only minimally displaced supracondylar humeral fractures that had been treated with cast immobilisation were considered. The values obtained for the Baumann angle of the humerus in this study were provided only as a reference to describe the differences between the different observations. In order to determine the normal values of the Baumann angle of the humerus in the paediatric population, a much larger study would be required, controlling for systematic errors that could affect the measurement of the angle (e.g. type and severity of fracture, quality of fracture reduction, the amount of rotation of the humerus on the radiograph, etc). The fact that these factors were not controlled in this study does not affect the results obtained, since the same radiographs were analysed by all observers.

In conclusion, the Baumann angle of the humerus is a simple, repeatable and reliable measurement that can be used for the determination of the outcome of supracondylar humeral fractures in the paediatric population.

Open Access This article is distributed under the terms of the Creative Commons Attribution Noncommercial License which permits any noncommercial use, distribution, and reproduction in any medium, provided the original author(s) and source are credited.

\section{References}

1. Cheng JC, Lam TP, Shen WY (1995) Closed reduction and percutaneous pinning for type III displaced supracondylar fractures of the humerus in children. J Orthop Trauma 9:511-515

2. Omid R, Choi PD, Skaggs DL (2008) Supracondylar humeral fractures in children. J Bone Jt Surg Am 90:1121-1132

3. Otsuka NY, Kasser JR (1997) Supracondylar fractures of the humerus in children. J Am Acad Orthop Surg 5:19-26

4. Camp J, Ishizue K, Gomez M, Gelberman R, Akeson W (1993) Alteration of Baumann's angle by humeral position: implications for treatment of supracondylar humerus fractures. J Pediatr Orthop 13:521-525

5. Skaggs DL, Cluck MW, Mostofi A, Flynn JM, Kay RM (2004) Lateral-entry pin fixation in the management of supracondylar fractures in children. J Bone Jt Surg Am 86-A:702-707

6. Acton JD, McNally MA (2001) Baumann's confusing legacy. Injury $32: 41-43$

7. Williamson DM, Coates CJ, Miller RK, Cole WG (1992) Normal characteristics of the Baumann (humerocapitellar) angle: an aid in assessment of supracondylar fractures. J Pediatr Orthop 12:636639

8. Keenan WN, Clegg J (1996) Variation of Baumann's angle with age, sex, and side: implications for its use in radiological monitoring of supracondylar fracture of the humerus in children. J Pediatr Orthop 16:97-98

9. France J, Strong M (1992) Deformity and function in supracondylar fractures of the humerus in children variously treated by closed reduction and splinting, traction, and percutaneous pinning. J Pediatr Orthop 12:494-498

10. Kallio PE, Foster BK, Paterson DC (1992) Difficult supracondylar elbow fractures in children: analysis of percutaneous pinning technique. J Pediatr Orthop 12:11-15

11. Kocher MS, Kasser JR, Waters PM, Bae D, Snyder BD, Hresko MT, Hedequist D, Karlin L, Kim YJ, Murray MM, Millis MB, Emans JB, Dichtel L, Matheney T, Lee BM (2007) Lateral entry compared with medial and lateral entry pin fixation for completely displaced supracondylar humeral fractures in children. A randomized clinical trial. J Bone Jt Surg Am 89:706-712 
12. Nacht JL, Ecker ML, Chung SM, Lotke PA, Das M (1983) Supracondylar fractures of the humerus in children treated by closed reduction and percutaneous pinning. Clin Orthop Relat Res 177:203-209

13. Pirone AM, Graham HK, Krajbich JI (1988) Management of displaced extension-type supracondylar fractures of the humerus in children. J Bone Jt Surg Am 70:641-650
14. Reitman RD, Waters P, Millis M (2001) Open reduction and internal fixation for supracondylar humerus fractures in children. $\mathrm{J}$ Pediatr Orthop 21:157-161

15. Skaggs DL, Hale JM, Bassett J, Kaminsky C, Kay RM, Tolo VT (2001) Operative treatment of supracondylar fractures of the humerus in children. The consequences of pin placement. J Bone Joint Surg Am 83-A:735-740 